INTERVIEW

For reprint orders, please contact: reprints@futuremedicine.com

\title{
Interview with VolitionRX on their nucleosomics blood tests for the detection of colorectal cancer
}

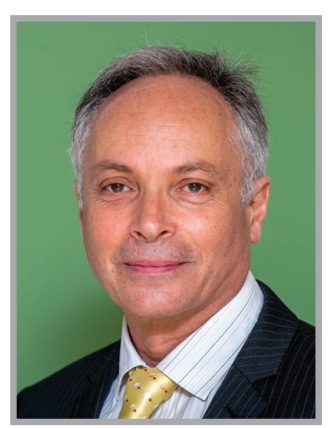

Jake Micallef* speaks to Caroline Telfer, Commissioning Editor: Dr Micallef has 20 years of experience in research and development and in the management of early-stage biotechnical companies, including the manufacture of biotechnology products and the establishment of manufacturing operations. Dr Micallef was educated at King's College London (BSc in biology and chemistry, 1977 and PhD in physical chemistry, 1981); St Thomas' Hospital Medical School, London (MSc in chemical pathology, 1985); and Imperial College Management School (MBA, 2000). He spent 10 years working at the WHO where he worked on new diagnostic products in the area of reproductive health and cancer.

Q Can you tell us a little about your career background?

I was originally educated as a physical chemist, but have dedicated almost my entire career to the development of ELISA-type blood tests in all sorts of diseases, including cancer. I started my career with the National Health Service during the 1980s where I developed, produced and distributed tests for reproductive steroids that were used in hospitals all over the UK. I then worked for the WHO for more than a decade where I led the development and manufacture of tests in reproductive health, cancer, thyroid disease and other indications that were used right across the whole world. Following a year out to study for an MBA, I worked in a small drug development company before joining VolitionRx at their research laboratories in Namur, Belgium in 2010.

Q What sparked your interest in cancer diagnostic technologies?
I have always had an interest in cancer diagnostics. When I originally came across the idea for the nucleosomics technology, I immediately thought this could be the ultimate in ELISA cancer diagnostics.

Q VolitionRx have recently announced the commencement of a large clinical trial to test the accuracy of the Nucleosome Quantification assay. Why is there a need for blood-based diagnostic tests for colorectal cancer? Colorectal cancer (CRC) is the second most common cause of cancer death in the UK after lung cancer. The mortality rate has fallen in the UK with the introduction of CRC fecal screening tests for people aged over 60 years, but less than half of all cancers are detected because only approximately half of the people sent a fecal test actually do it. This is because three fecal samples must be taken following 3 days of pretest preparation and some people find

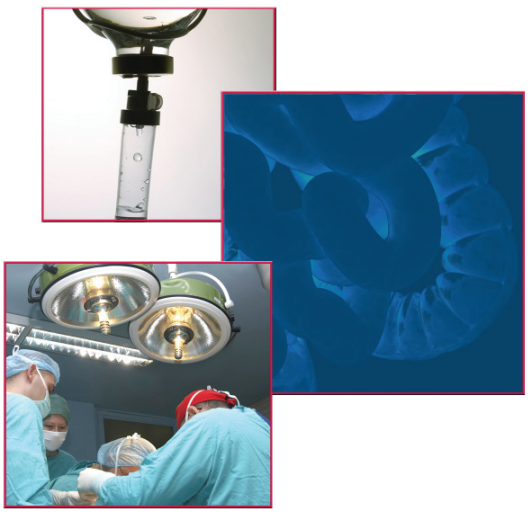

News \& Views

News

Interview

"Colorectal cancer can be treated effectively if diagnosed early, before it becomes aggressive and spreads to other parts of the body."

*VolitionRX, Centre Technologique, Rue duSéminaire 20A, B-5000 Namur, Belgium; j.micallef@volitionrx.com

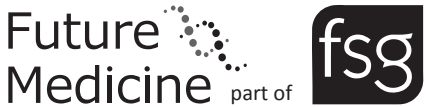


fecal sampling distasteful. Research, including our own survey, shows that up to $97 \%$ of people will take a blood test so there is a potential for a great improvement in CRC detection rate and survival rates with a CRC blood test.

\section{Q Can you explain a little about the theory} behind the Nucleosome Quantification assay?

$\mathrm{NuQ}$ is short for nucleosome quantification. Our cells contain the chromosomes that contain our DNA and genes. These chromosomes are made up of nucleosomes (in the same way that starch is made of glucose). Each of our 46 chromosomes is essentially a coiled up string of about half a million nucleosomes arranged like beads on a string where the string is the DNA, as shown in Figure 1.

Volition's NuQ assays represent a combination of two independent lines of cancer research that have each been going on since the year 2000. The first line has shown that the chromosomes and nucleosomes in cancer cells have different chemical structures to those in healthy people. The second has shown that nucleosomes from dead cancer cells are present in the blood of cancer patients. Our NuQ tests detect these altered nucleosomes in the blood of cancer patients to detect the cancer.

\section{Q There have been some preliminary} studies conducted in Belgium and Denmark using the NuQ assays. Can you describe the results \& implications of these studies?

Our early pilot studies on small numbers of patients in Belgium have shown that $\mathrm{NuQ}$ assays are able to detect colon cancer and discriminate between people with colon cancer and healthy people. NuQ assays can also distinguish between people with colon cancer and people

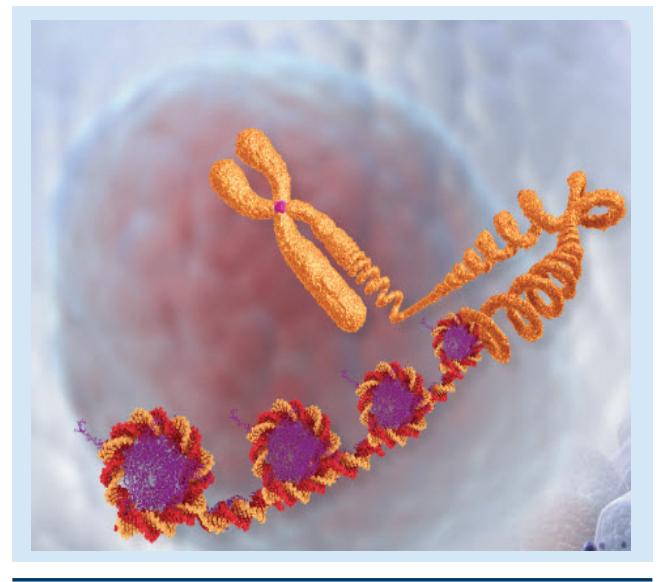

Figure 1. Nucleosomes in cellular DNA. with other colon diseases (Crohn's disease, colitis and diverticulosis among others).

Our early Belgian studies have also shown that $\mathrm{NuQ}$ assays are able to detect prostate cancer and - most exciting of all - to distinguish between people with prostate cancer and colon cancer. To have proof that with the right panel combination, $\mathrm{NuQ}$ assays can distinguish between different types of cancer as well as healthy samples, is just what we have been working towards. CRC and prostate cancer may be just the start.

We are now part way through a much larger blind retrospective study of 4800 patients referred for colonoscopy at the Hvidovre Hospital (Copenhagen, Denmark) to confirm our findings in the Belgian studies. We have also signed up to an 11,000 prospective colon cancer screening trial with Hvidovre Hospital, which is commencing next month.

\section{Q Can you explain the details of the new} large clinical trial that has just commenced? The new blind clinical study is designed to confirm the findings of the Belgian colon cancer study in a much larger number of patients -4800 symptomatic patients referred for colonoscopy at the Hvidovre Hospital. If successful, this will provide incontrovertible evidence of the clinical utility of $\mathrm{NuQ}$ assays in CRC and will be used as part of the necessary evidence for regulatory approval for the assays in Europe.

\section{Q What were the entry criteria for patients to be included in the study?}

The patients all displayed symptoms that indicated they may have CRC. For this reason, they were referred for colonoscopy and were asked if they would participate in the study.

\section{Q What results do you expect to see in this trial?}

I expect the results will confirm the findings of our early pilot studies conducted in Belgium.

Q What are the implications of the results of this trial? What do you think will be the next steps for the NuQ assay?

There have been no significant new diagnostic cancer blood tests in more than 25 years. I expect the results of our study will demonstrate the ground-breaking utility of $\mathrm{NuQ}$ assays. The next step for the NuQ assays will be to obtain regulatory approval for their diagnostic use in Europe. 
Q How do you think the introduction of this technology will change clinical practice in this area?

CRC can be treated effectively if diagnosed early, before it becomes aggressive and spreads to other parts of the body. Unfortunately, cancer is currently often detected late when treatments are much less effective. I think $\mathrm{NuQ}$ assays will improve cancer detection and, therefore, the outcome for patients, while saving money for the National Health Service by reducing the requirement for the very expensive drugs used to treat late-stage cancer patients.

\section{Disclaimer}

The opinions expressed in this interview are those of the interviewee and do not necessarily reflect the views of Future Medicine Ltd.

\section{Financial \& competing interests disclosure}

$J$ Micallef is a stockholder and a paid consultant to VolitionRX. J Micallef has no other relevant affiliations or financial involvement with any organization or entity with a financial interest in or financial conflict with the subject matter or materials discussed in the manuscript apart from those disclosed.

No writing assistance was utilized in the production of this manuscript. 